ITP-SB-95-54

hep-th/9512075

December 1995

\title{
Supersymmetric $\sigma$-models, twistors, and the Atiyah-Hitchin metric
}

\author{
Ivan T. Ivanovfl and M. Ročekf \\ Institute for Theoretical Physics \\ State University of New York \\ Stony Brook, NY 11794-3840 USA
}

\begin{abstract}
The Legendre transform and its generalizations, originally found in supersymmetric $\sigma$-models, are techniques that can be used to give local constructions of hyperkähler metrics. We give a twistor space interpretation to the generalizations of the Legendre transform construction. The Atiyah-Hitchin metric on the moduli space of two monopoles is used as a detailed example.
\end{abstract}

\footnotetext{
${ }^{1}$ email: iti@insti.physics.sunysb.edu

${ }^{2}$ email: rocek@insti.physics.sunysb.edu
} 


\section{Introduction.}

Some time ago, we discoverd several constructions of hyperkähler metrics by studying extended supersymmetric nonlinear sigma-models [1, 2, 3, 4]. In the hyperkähler quotient framework, the topology of the manifold and the completeness of the metric are accessible. On the other hand, the Legendre transform construction and its generalizations are purely local. They can be considered as changes of variables that linearize the Monge-Ampère equations governing hyperkähler metrics. In some cases, the Legendre transform construction has been used to find complete metrics. A twistor space interpretation has also been worked out. In this paper, we consider the simplest generalization of the Legendre transform construction [4]. We reformulate it using twistors and empasize the special properties of the twistor spaces used. Throughout this paper we consider only the four dimensional case to avoid congesting the notation, but all the techniques are easily generalized to higher dimensions. As the main example we show that the hyperkähler metric in the moduli space of two monopoles [7] can be obtained by a generalized Legendre transform construction from a single simple function. Using this example we also propose various ingredients in the construction of new complete metrics.

In section two we introduce the generalized Legendre transform construction from 4 . Section three contains a brief and, we hope, readable outline of the basics of twistor space. In section four, the twistor interpretation of the generalized Legendre transform construction is worked out. A careful treatment of the singularities of the functions involved is essential. Section five briefly reconsiders the twistor construction of $A_{k} A L E$-metrics from a new point of view; in particular, the singularities that arise in this construction, which were regarded in [2] as something of an embarrassment, are understood and shown to play a crucial role. Section six is devoted to the Atiyah-Hitchin metric in the framework of the generalized Legendre transform construction. In the final section we notice that many of the complete metrics we have discussed in the paper are built from a few "flat space" ingredients. We propose more complete metrics based on these. 


\section{Generalized Legendre transform construc- tion.}

To set the stage we review the generalized Legendre transform construction of hyperkähler metrics [4]. Consider $\eta(\zeta)=\sum_{a=0}^{2 n} w_{a} \zeta^{a}$ to be a polynomial of order $2 n$ in $\zeta$, where $\zeta$ is the coordinate on the Riemann sphere $\mathbf{C P}_{1}=S^{2}$. This polynomial should obey a reality condition: $\overline{\eta(\zeta)}=(-1)^{n} \bar{\zeta}^{2 n} \eta(-1 / \bar{\zeta})$, which implies $w_{2 n-a}=(-1)^{n+a} \bar{w}_{a}$. The Kähler potential is constructed from one function of two variables $G(\eta(\zeta), \zeta)$. Consider the function $F$

$$
F\left(w_{a}\right)=\frac{1}{2 \pi i} \oint_{C} \frac{d \zeta}{\zeta^{2}} G(\eta, \zeta)
$$

where $C$ is some appropriately chosen contour. $F$ obeys a system of linear differential equations

$$
F_{a b}=F_{a+c, b-c}, \text { where } F_{a b} \equiv \frac{\partial^{2} F}{\partial w_{a} \partial w_{b}} .
$$

Let $z=w_{0}, v=w_{1}$; then $w_{2 n}=(-1)^{n} \bar{z}$ and $w_{2 n-1}=-(-1)^{n} \bar{v}$. The Kähler potential is constructed from the function $F\left(w_{a}\right)$ by performing a two dimensional Legendre transform with respect to $v$ and $\bar{v}$, and extremizing with respect to the remaining coordinates $\left\{w_{a^{\prime}}, 2 \leq a^{\prime} \leq 2 n-2\right\}$.

$$
K(z, \bar{z}, u, \bar{u})=F\left(z, \bar{z}, v, \bar{v}, w_{a^{\prime}}\right)-u v-\bar{u} \bar{v},
$$

where

$$
v=v(z, \bar{z}, u, \bar{u}), w_{a^{\prime}}=w_{a^{\prime}}(z, \bar{z}, u, \bar{u})
$$

are solutions to the equations

$$
F_{v}=u, F_{a^{\prime}}=0,2 \leq a^{\prime} \leq 2 n-2 .
$$

The metric is obtained as usual from the Kähler potential and can be expressed in terms of second derivatives of the function $F\left(w_{a}\right)$. We refer to [4] for the explicit equations. 


\section{Brief review of twistor approach.}

We start with very brief review of twistor theory for hyperkähler manifolds (for details and proofs see [2]). A hyperkähler manifold $\mathbf{M}$ is a Riemannian manifold which is Kähler with respect to three complex structures $\mathbf{I}, \mathbf{J}$, and $\mathbf{K}$ that obey the quaternionic algebra relations

$$
\mathbf{I}^{2}=\mathbf{J}^{2}=\mathbf{K}^{2}=-1, \mathbf{I J}=-\mathbf{J I}=\mathbf{K}, \text { etc. }
$$

The Kählerian conditions imply that the metric $g$ on $\mathbf{M}$ is hermitian with respect to the complex structures and the complex structures are covariantly constant with respect to the Levi-Civita connection: for arbitrary vector fields $\mathbf{X}, \mathbf{Y}$

$$
g(\mathbf{I X}, \mathbf{I Y})=g(\mathbf{X}, \mathbf{Y}), \nabla \mathbf{I}=0, \text { etc. }
$$

Clearly, if $(a, b, c)$ is a unit vector in $\mathbf{R}^{3}$, the properties of $\mathbf{I}, \mathbf{J}$, and $\mathbf{K}$ imply that $\mathbf{I}(a, b, c)=a \mathbf{I}+b \mathbf{J}+c \mathbf{K}$ is another complex structure compatible with the metric and the connection. Thus, on a hyperkähler manifold, there is a two-sphere's worth of Kähler structures. The twistor space $\mathbf{T}$ of the hyperkähler manifold $\mathbf{M}$ is then constructed so as to incorporate all the complex structures together on a larger complex manifold [2].

As a manifold the twistor space $\mathbf{T}$ is just the product $\mathbf{M} \times S^{2}$. Accordingly, the tangent space to $\mathbf{T}$ splits as the direct sum $\mathbf{T}_{m} \oplus \mathbf{T}_{\zeta}$. To see that $\mathbf{T}$ is a complex manifold, we first consider a complex structure on $S^{2}$. The Riemann sphere $S^{2}$ considered as the complex projective line $\mathbf{C P}_{1}$ is a complex manifold obtained by patching together two copies $U, \tilde{U}$ of the complex plane $\mathbf{C}$ with coordinates $\zeta, \tilde{\zeta}$ related by $\tilde{\zeta}=\zeta^{-1}$ on $U \cap \tilde{U}$. We will use this model of $\mathbf{C P}_{1}$ throughout the paper and refer to $\zeta$ as the standard coordinate on it. Define a almost complex structure $\mathbf{I}(\zeta)$ on $\mathbf{T}$ by

$$
\underline{\mathbf{I}}=\left(\mathbf{I}(\zeta), I_{0}\right)=\left(\frac{1-\zeta \bar{\zeta}}{1+\zeta \bar{\zeta}} \mathbf{I}+\frac{\zeta+\bar{\zeta}}{1+\zeta \bar{\zeta}} \mathbf{J}+i \frac{\zeta-\bar{\zeta}}{1+\zeta \bar{\zeta}} \mathbf{K}, I_{0}\right)
$$

Here the two operators $\mathbf{I}(\zeta)$ and $I_{0}$ act on $\mathbf{T}_{m}$ and $\mathbf{T}_{\zeta}$ respectively. $I_{0}$ is the standard complex structure on $\mathbf{C P}_{1}$ as described above.

It is easy to prove that $\mathbf{I}$ is an integrable almost complex structure and so $\mathbf{T}$ is a complex manifold and admits complex coordinates. Moreover the 
projection $\mathbf{T} \rightarrow \mathbf{C P}_{1}$ is holomorphic and each copy $\left(m, \mathbf{C P}_{1}\right)$ (where $m$ is a point on $\mathbf{M}$ ) of the projective line is a holomorphic section of this projection. These sections are called twistor lines.

Another key ingredient in the twistor space approach is a certain holomorphic symplectic form. For a Kähler manifold the metric $g$ and the complex structure $\mathbf{J}$ can be combined into a symplectic form $\omega(\mathbf{X}, \mathbf{Y}) \equiv g(\mathbf{J X}, \mathbf{Y})$ called the Kähler form. For a hyperkähler manifold, there are three Kähler forms $\omega_{1}, \omega_{2}$, and $\omega_{3}$ corresponding to $\mathbf{I}, \mathbf{J}$, and $\mathbf{K}$ respectively. Let $z_{1}$ and $z_{2}$ be (local) holomorphic coordinates on $\mathbf{M}$ with respect to the complex structure $\mathbf{I}$, i.e., $\mathbf{I} d z_{1}=i d z_{1}, \mathbf{I} d z_{2}=i d z_{2}$. It is easy to prove that one can choose the coordinates $z_{1}, z_{2}$ such that $d z_{1} \wedge d z_{2}=\left(\omega_{2}+i \omega_{3}\right) / 2$. Thus the two-form $\omega_{+}=\left(\omega_{2}+i \omega_{3}\right) / 2$ is a holomorphic $(2,0)$-form with respect to the complex structure $\mathbf{I}$. One can extend $\omega_{+}$to a holomorphic form on $\mathbf{T}$ by defining:

$$
\omega_{h}(\zeta)(\mathbf{X}, \mathbf{Y}) \equiv \omega_{+}(\mathbf{X}+\zeta \mathbf{K X}, \mathbf{Y}+\zeta \mathbf{K Y})
$$

The Clifford algebra of the complex stuctures guarantees that $\omega_{h}(\zeta)$ is holomorphic $(2,0)$-form for $\mathbf{I}(\zeta)$ and can be written out as

$$
2 \omega_{h}(\zeta)=\left(\omega_{2}+i \omega_{3}\right)+2 \zeta \omega_{1}-\zeta^{2}\left(\omega_{2}-i \omega_{3}\right) .
$$

Note that $\omega_{h}(\zeta)$ is quadratic in $\zeta$; this is expressed mathematically by saying that $\omega_{h}(\zeta)$ is section of $\Lambda^{2} \mathbf{M}$ (i.e., a 2-form) twisted by $\mathcal{O}(2)$. Here $\mathcal{O}(2)$ is the line bundle over $\mathbf{C P}_{1}$ of degree two. In general, by $\mathcal{O}(n)$ we denote the line bundle over $\mathbf{C P}_{1}$ of degree $n$. If $\zeta$ is the standard coordinate on $\mathbf{C P}_{1}$, the sections of the bundle $\mathcal{O}(n)$ can be identified with polynomials of degree $n: a_{n} \zeta^{n}+\cdots+a_{1} \zeta+a_{0}$.

The last ingredient in the twistor space picture is a map $\tau: \mathbf{M} \times S^{2} \rightarrow$ $\mathbf{M} \times S^{2}$ called a real structure. It is defined by

$$
\tau(m, \zeta)=\left(m,-\frac{1}{\bar{\zeta}}\right)
$$

Thus $\tau$ is the antipodal map on the $S^{2}$ factor, and from (18) it takes the complex structure $\underline{\mathbf{I}}$ to its conjugate $-\underline{\mathbf{I}}$. It codes ordinary complex conjugation. All the holomorphic data given is compatible with the real structure composed with complex conjugation (see, e.g., eq. 13).

This construction is invertible, and given a twistor space with all the properties above, we can reconstruct $\mathbf{M}$ and the hyperkähler metric on it. 
We will not go into any further details, and merely refer the interested reader to [2] and [5].

In practice it is quite difficult to obtain hyperkähler metrics from twistor spaces even if the data needed on them is essentially free, because some steps in the inverse construction (in particular finding the twistor lines) are quite involved. The relatively easy case of hyperkähler metrics with triholomorphic isometries is treated thoroughly in [2] (see also [6]) and sometimes referred to as the Legendre transform construction. In the next section we present a generalization that works for metrics with special twistor spaces.

\section{Twistor point of view on the generalized Legendre transform construction.}

By construction, the twistor space $\mathbf{T}$ of the hyperkähler manifold $\mathbf{M}$ has a projection onto $\mathbf{C P}_{1}$, and the fibers of this bundle are copies of $\mathbf{M}$. We consider twistor spaces which also have (intermediate) holomorphic projections onto line bundles over $\mathbf{C P}_{1}$ of positive even degree.

$$
\mathbf{T} \rightarrow \mathcal{O}(2 n) \rightarrow \mathbf{C P}_{1}
$$

This requirement is equivalent to the existence of a $\mathbf{I}(\zeta)$-holomorphic coordinate $\eta(\zeta)$ on $\mathbf{M}$, such that $\eta(\zeta)$ is polynomial of order $2 n$. An additional condition we impose is that $\eta(\zeta)$ is real under the real structure $\tau$

$$
\overline{\eta(\zeta)}=(-1)^{n} \bar{\zeta}^{2 n} \eta(-1 / \bar{\zeta})
$$

The case $n=1$ applies to metrics with triholomorphic isometry.

Our model of $\mathbf{C P}_{1}$ was build by two patches $U$ and $\tilde{U}$ with local coordinates $\zeta$ and $\tilde{\zeta}$ related by $\tilde{\zeta}=\zeta^{-1}$ on the intersection $U \cap \tilde{U}$. The polynomial expression for the complex coordinate $\eta(\zeta)=\sum_{a=0}^{2 n} w_{a} \zeta^{a}$ is good only on the patch $U$. On $\tilde{U}$ the coordinate is $\tilde{\eta}\left(\zeta^{-1}\right)$ and on $U \cap \tilde{U}$ the two are related by

$$
\tilde{\eta}=\zeta^{-2 n} \eta
$$

Let $\chi(\zeta)(\tilde{\chi}(\zeta))$ be the second holomorphic coordinate on $\mathbf{M}$ over $U(\tilde{U})$, respectively. The holomorphic form $\omega_{h}(\zeta)$ can be evaluated and compared on the two patches

$$
\omega_{h}=d \eta \wedge d \chi=\zeta^{2} d \tilde{\eta} \wedge d \tilde{\chi}
$$


The second equality follows because $\omega_{h}$ is a two form twisted by $\mathcal{O}(2)$. From (14) and (15) we see that the expressions for the second holomorphic coordinate on the two patches $U$ and $\tilde{U}$ are connected by

$$
\tilde{\chi}=\zeta^{2 n-2} \chi-f(\eta, \zeta)
$$

Here $f(\eta, \zeta)$ is arbitrary function of $\eta$ and $\zeta$. Even if $f(\eta, \zeta)=0$, we can not conclude that $\chi(\zeta)$ is a section of $\mathcal{O}(2-2 n)$, since it can have poles or cuts or both as a function of $\zeta$. As we shall see, the singularities of $\chi(\zeta)$ play a crucial important role in determining the metric on $\mathbf{M}$.

We now expand the coordinates $\eta(\zeta)$ and $\chi(\zeta)$ in Taylor series around the point $\zeta=0 . \rho^{\text {P }}$

$$
\begin{aligned}
& \eta(\zeta)=z+v \zeta+w_{2} \zeta^{2}+\cdots+ \\
& \quad+w_{2 n-2} \zeta^{2 n-2}+(-1)^{n-1} \bar{v} \zeta^{2 n-1}+(-1)^{n} \bar{z} \zeta^{2 n} \\
& \chi(\zeta)=u+t \zeta+O\left(\zeta^{2}\right) .
\end{aligned}
$$

Note that the particular expansion of $\eta(\zeta)$ is guided by the reality condition (13), and $z$ and $u$ are complex coordinates for the complex structure at $\zeta=0$, namely I. The expansion of $\tilde{\eta}(\zeta)$ around $\zeta=\infty$ follows from (14) and for the expansion of $\tilde{\chi}$ we write

$$
\tilde{\chi}\left(\zeta^{-1}\right)=\tilde{u}+\tilde{t} \zeta^{-1}+O\left(\zeta^{-2}\right) .
$$

Next, following [2], we compare the coefficients for different powers of $\zeta$ in (16). Consider a integral along a small contour around the point $\zeta=\infty$ of both sides of equation (16)

$$
\frac{1}{2 \pi i} \oint_{\infty} \frac{d \zeta}{\zeta^{m}} \tilde{\chi}=\frac{1}{2 \pi i} \oint_{\infty} \frac{d \zeta}{\zeta^{m+2-2 n}} \chi-\frac{1}{2 \pi i} \oint_{\infty} \frac{d \zeta}{\zeta^{m}} f(\eta, \zeta) .
$$

The integral on the left hand side will pick up the appropriate coefficient in the Taylor expansion. To select the appropriate coefficient of the $\chi$ expansion, however, the contour should be deformed to a contour around $\zeta=0$. In the process we will pick contributions from the singularities of $\chi$ at points $\zeta \neq \infty$. We assume that the corresponding terms can be expressed in terms

\footnotetext{
${ }^{3}$ We assume that the singularities of $\chi$ are away from $\zeta=0$.
} 
of a contour integral of a function $f^{\prime}(\eta, \zeta)$. This assumption is satisfied for a host of examples and amounts to saying that the twistor lines are specified by a choice of the coefficients of $\eta$. Thus equation (20) can be rewritten as

$$
\begin{aligned}
\frac{1}{2 \pi i} \oint_{\infty} \frac{d \zeta}{\zeta^{m}} \tilde{\chi}=\frac{1}{2 \pi i} \oint_{0} \frac{d \zeta}{\zeta^{m+2-2 n}} \chi & -\frac{1}{2 \pi i} \oint_{C} \frac{d \zeta}{\zeta^{m}} f^{\prime}(\eta, \zeta) \\
& -\frac{1}{2 \pi i} \oint_{\infty} \frac{d \zeta}{\zeta^{m}} f(\eta, \zeta) .
\end{aligned}
$$

To make the notation more compact we combine the last two terms into one integral of a new function $\hat{f}(\eta, \zeta)$. For every particular example it will be clear which is the contribution coming from the singularities of $\chi$, and what kind of contour is to be taken around these singularities.

$$
\frac{1}{2 \pi i} \oint_{\infty} \frac{d \zeta}{\zeta^{m}} \tilde{\chi}=\frac{1}{2 \pi i} \oint_{0} \frac{d \zeta}{\zeta^{m+2-2 n}} \chi-\frac{1}{2 \pi i} \oint_{C} \frac{d \zeta}{\zeta^{m}} \hat{f}(\eta, \zeta) .
$$

It is convenient to intoduce a new function $G(\eta, \zeta)$ such that $\partial G / \partial \eta=$ $\zeta^{2-2 n} \hat{f}$, as well as the following integral of $G$

$$
F=\frac{1}{2 \pi i} \oint_{C} \frac{d \zeta}{\zeta^{2}} G(\eta, \zeta)
$$

Here $F$ is a function of the coefficients of $\eta(\zeta)$, and is precisely the function of section two. We also have

$$
F_{w_{j}}=\frac{1}{2 \pi i} \oint_{C} \frac{d \zeta}{\zeta^{2 n-j}} \hat{f}(\eta, \zeta)
$$

A comparison with (22) and the expansion series shows that $F_{w_{a^{\prime}}}=0$ for $2 \leq$ $a^{\prime} \leq 2 n-2, F_{z}=t, \quad F_{v}=u, F_{\bar{v}}=(-1)^{n} \tilde{u}, F_{\bar{z}}=(-1)^{n+1} \tilde{t}$. For the Kähler potential to be real the function $F$ should be real and it follows that $\tilde{t}=(-1)^{n+1} \bar{t}, \tilde{u}=(-1)^{n} \bar{u}$, etc.

From (10), the Kähler form is the coefficient of $\zeta$ in the expansion of the $\mathbf{I}(\zeta)$ holomorphic form $\omega_{h}$, and (15) then implies

$$
\omega_{1}=d z \wedge d t+d v \wedge d u=d z \wedge d\left(F_{z}\right)+d v \wedge d u .
$$


Defining a new function

$$
K(z, \bar{z}, u, \bar{u})=F\left(z, \bar{z}, v, \bar{v}, w_{a^{\prime}}\right)-u v-\bar{u} \bar{v}
$$

and taking into account the relations already inferred,

$$
F_{v}=u, \quad F_{\bar{v}}=\bar{u}, \text { and } F_{w_{a^{\prime}}}=0 ; 2 \leq a \leq 2 n-2,
$$

we derive the following relations

$$
\begin{aligned}
K_{z} & =F_{z}+F_{v} \frac{\partial v}{\partial z}+F_{\bar{v}} \frac{\partial \bar{v}}{\partial z}+F_{w_{a^{\prime}}} \frac{\partial w_{a^{\prime}}}{\partial z}-u \frac{\partial v}{\partial z}-\bar{u} \frac{\partial \bar{v}}{\partial z}=F_{z} \\
d v & =-d K_{u} .
\end{aligned}
$$

Substitution into (25) gives

$$
\begin{aligned}
\omega_{1} & =d z \wedge d\left(K_{z}\right)-d\left(K_{u}\right) \wedge d u= \\
& =K_{z \bar{z}} d z \wedge d \bar{z}+K_{z \bar{u}} d z \wedge d \bar{u}+K_{u \bar{z}} d u \wedge d \bar{z}+K_{u \bar{u}} d u \wedge d \bar{u}
\end{aligned}
$$

and so $\omega_{1}$ is the Kähler form for a metric with Kähler potential $K$ (26). This ends our formal construction, and explains the generalized Legendre transform construction in a twistor space setting. We now consider examples.

\section{$5 \quad A_{k} A L E$ metrics reconsidered}

As a first illustration of the above construction, we reinterpret the twistor space construction of the $A_{k}$ asymptotically locally euclidean metrics [8] by the Legendre transform. The twistor space $\mathbf{T}$ of the $A_{k} A L E$ metrics can be described as the zero locust of the following polynomial:

$$
x(\zeta) y(\zeta)-\prod_{i=1}^{k+1}\left(\eta(\zeta)-a_{i}(\zeta)\right)=0
$$

where,

$$
x(\zeta) \in \Gamma(\mathcal{O}(k+1)), \quad y(\zeta) \in \Gamma(\mathcal{O}(k+1)), \quad \eta(\zeta) \in \Gamma(\mathcal{O}(2)),
$$

\footnotetext{
${ }^{4}$ This is true up to resolution of some singularities, but this subtlety will not influence our discussion.
} 
$a_{i}$ are fixed real (with respect to the real structure) sections of $\mathcal{O}(2)$ that parametrize the moduli space of metrics, $\eta$ is also real and its coefficients are determined by the coordinates on the manifold. The real structure exchanges $x$ and $y$. We write $\eta$ and $a_{i}$ explicitly as:

$$
\eta=z+v \zeta-\bar{z} \zeta^{2}, \quad a_{i}=p_{i}+q_{i} \zeta-\bar{p}_{i} \zeta^{2}
$$

Guided by the real structure, Hitchin [8] solves (31) as follows. Let

$$
\begin{gathered}
\eta-a_{i}=\left(-\bar{z}+\bar{p}_{i}\right)\left(\zeta-\alpha_{i}\right)\left(\zeta+1 / \bar{\alpha}_{i}\right), \\
\alpha_{i}=\frac{\left(v-q_{i}\right)-\sqrt{\left(v-q_{i}\right)^{2}+4\left|z-p_{i}\right|^{2}}}{2\left(\bar{z}-\bar{p}_{i}\right)} .
\end{gathered}
$$

Then set

$$
x=A \prod_{i=1}^{k+1}\left(\zeta-\alpha_{i}\right), \quad y=B \prod_{i=1}^{k+1}\left(\zeta+1 / \bar{\alpha}_{i}\right)
$$

where $A B=\prod\left(-\bar{z}+\bar{p}_{i}\right)$ and $A \bar{A}=\prod\left(\left(v-q_{i}\right)+\sqrt{\left(v-q_{i}\right)^{2}+4\left|z-p_{i}\right|^{2}}\right)$.

The holomorphic form is given by

$$
\omega_{h}=d \eta \wedge\left(\frac{d x}{x}-\frac{d y}{y}\right) .
$$

Thus the second $\mathbf{I}(\zeta)$-holomorphic coordinate is

$$
\chi=\ln \left(\frac{x}{y}\right)
$$

These considerations are all valid on the patch $\zeta \in U$. The corresponding quantities on the other patch $\tilde{U}$ are

$$
\tilde{\eta}=\zeta^{-2} \eta, \quad \tilde{x}=\zeta^{-(k+1)} x, \quad \tilde{y}=\zeta^{-(k+1)} y .
$$

These relations imply

$$
\tilde{\chi}=\chi
$$


and the function $f(\eta, \zeta)$ in (16) vanishes. By comparing the expansions of $\chi$ and $\tilde{\chi}$ as in (22) we read off the function $\hat{f}(\eta, \zeta)$ and the contour of integration

$$
\oint_{C} \frac{d \zeta}{\zeta^{m}} \hat{f}(\eta, \zeta)=\left(\oint_{0}-\oint_{\infty}\right) \frac{d \zeta}{\zeta^{m}} \ln \left(\frac{x}{y}\right) .
$$

The pairs of zeroes of $\eta-a_{i}$ are zeroes of $x$ and $y$ in the logarithm in the right hand side above, and the two contours around 0 and $\infty$ can be deformed to $k+1$ contours around the pairs of roots of $\eta-a_{i}$. Equation (41) can be rewritten as

$$
\oint_{C} \frac{d \zeta}{\zeta^{m}} \hat{f}(\eta, \zeta)=\oint_{C} \frac{d \zeta}{\zeta^{m}} \sum_{i=1}^{k+1} \ln \left(\eta-a_{i}\right)
$$

where the contour $C$ circles each root with the opposite orientation (after using the identity in Figure 1). Integrating $\hat{f}(\eta, \zeta)$ once with respect to $\eta$ we

$$
\begin{aligned}
& C_{1} \text { : } \quad C_{2} \text { : } \\
& \stackrel{\bullet}{\longrightarrow} \\
& \oint_{C_{1}} d \zeta f(\zeta) \ln \left(\frac{\zeta-a}{\zeta-b}\right)=\oint_{C_{2}} d \zeta f(\zeta) \ln ((\zeta-a)(\zeta-b))
\end{aligned}
$$

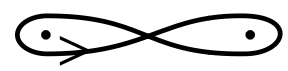

Figure 1: An identity between integrals.

obtain the function $G(\eta, \zeta)$

$$
G(\eta, \zeta)=\sum_{i=1}^{k+1}\left(\eta-a_{i}\right)\left(\ln \left(\eta-a_{i}\right)-1\right),
$$

in agreement with [2]. For future reference, we also give the function $F$ for the Taub-NUT metric:

$$
F=\frac{1}{2 \pi i} \oint d \zeta\left(\frac{\eta^{2}}{\zeta^{3}}+m \frac{\eta(\ln \eta-1)}{\zeta^{2}}\right),
$$

where $m$ is called the mass parameter. 


\section{The Atiyah-Hitchin metric}

As another illustration of the generalized Legenedre transform construction, we consider the Atiyah-Hitchin metric on the moduli space of two centered $S U(2)$ monopoles. The metric is hyperkähler and known explicitely [7]. The twistor description is also known [7] and the twistor space has a holomorphic projection on the bundle $\mathcal{O}(4)$. Thus one of the complex coordinates of the metric has an expansion

$$
\eta(\zeta)=z+v \zeta+w \zeta^{2}-\bar{v} \zeta^{3}+\bar{z} \zeta^{4}
$$

Due to the reality condition, the roots of the quartic (45) come in two pairs of the type $\lambda,-1 / \bar{\lambda}$. It is convenient to write the quartic in terms of its roots and a scale factor $c$ :

$$
\eta(\zeta)=c(\zeta-\alpha)(\zeta-\beta)(\bar{\alpha} \zeta+1)(\bar{\beta} \zeta+1) .
$$

A starting point for the generalized Legendre transform construction is the function $G(\eta, \zeta)$. Asymptotically, the Atiyah-Hitchin metric approaches the Taub-NUT metric, and $\eta_{A H} \rightarrow \eta_{\text {Taub-NUT. }}^{2}$. Comparing to (44), we make the ansatz that the function $F$ for the Atiyah-Hitchin metric is given by

$$
F=-\frac{1}{2 \pi i} \oint_{0} \frac{d \zeta}{\zeta^{3}} \eta+\oint_{C} \frac{d \zeta}{\zeta^{2}} \sqrt{\eta}
$$

The contour $C$ encloses all four roots of $\eta$, and the branch cuts are chosen to run from $\alpha$ to $-1 / \bar{\beta}$ and from $\beta$ to $-1 / \bar{\alpha}$. The first step of the proof that (47) does indeed give the Atiyah-Hitchin metric is to solve the equation

$$
F_{w}=-1+\oint_{C} \frac{d \zeta}{\sqrt{\eta}}=0
$$

for $w$, or equivalently, to find the four parameter family of twistor lines inside the five real parameter family of quartics. The integral in (48) is a complete elliptic integral of the first kind; we transform it to Legendre normal form (10], p. 307).

$$
F_{w}=-1+\frac{4}{\sqrt{c(1+\alpha \bar{\alpha})(1+\beta \bar{\beta})}} \int_{0}^{1} \frac{d \zeta}{\sqrt{\left(1-\zeta^{2}\right)\left(1-k^{2} \zeta^{2}\right)}}=0
$$


where the modulus is

$$
k^{2}=\frac{(1+\alpha \bar{\beta})(1+\beta \bar{\alpha})}{(1+\alpha \bar{\alpha})(1+\beta \bar{\beta})} .
$$

It does not seem possible to solve for $w$ explicitly from (49), but instead we can solve for the scale in terms of the roots

$$
c=\frac{16 K^{2}(k)}{(1+\alpha \bar{\alpha})(1+\beta \bar{\beta})},
$$

where $K(k)$ is the Legendre complete integral of first kind. To make contact with the known form of the Atiyah-Hitchin metric we also reparametrize the roots of the quartic (or equivalently the coefficients) using new variables $\theta, \phi, \psi$, and then the restriction (48) on the coefficients of the quartic (45) is solved as follows

$$
\begin{aligned}
& z=2 \exp ^{2 i \phi}\left(\cos (2 \psi)\left(1+\cos ^{2}(\theta)\right)+\right. \\
& \left.+2 i \sin (2 \psi) \cos (\theta)+\left(2 k^{2}-1\right) \sin ^{2}(\theta)\right) K^{2}(k), \\
& v=8 \exp ^{i \phi} \sin (\theta)(\sin (2 \psi)- \\
& \left.-i \cos (2 \psi) \cos (\theta)+i\left(2 k^{2}-1\right) \cos (\theta)\right) K^{2}(k), \\
& w=4\left(-3 \cos (2 \psi) \sin ^{2}(\theta)+\left(2 k^{2}-1\right)\left(1-3 \cos ^{2}(\theta)\right)\right) K^{2}(k) .
\end{aligned}
$$

To check that (54) solves (51), we found the roots of the quartic with these coefficients; this also determines the scale, and then the direct substitution shows that (51) is satisfied. The expressions for the roots are quite long and not very illuminating, so we do not include them here. To clarify the particular form of the coefficients (54), notice that they and their complex conjugates $\bar{v}, \bar{z}$ form a five dimensional representation of the $S U(2)$ that rotates the sphere of complex structures (acts on $\zeta$ by fractional transformations). The vector fields generating this action have the standard form [11]:

$$
\begin{aligned}
& \mathcal{L}_{3}=-i \frac{\partial}{\partial \phi} \\
& \mathcal{L}_{+}=e^{i \phi}\left(i \frac{\partial}{\partial \theta}+\frac{1}{\sin (\theta)} \frac{\partial}{\partial \psi}-\cot (\theta) \frac{\partial}{\partial \phi}\right) \\
& \mathcal{L}_{-}=e^{-i \phi}\left(-i \frac{\partial}{\partial \theta}+\frac{1}{\sin (\theta)} \frac{\partial}{\partial \psi}-\cot (\theta) \frac{\partial}{\partial \phi}\right)
\end{aligned}
$$


Now the four variables $k, \theta, \phi, \psi$ select a four parameter family of quartics and parametrize the Atiyah-Hitchin manifold $\mathbf{M}$.

Next we can substitute the function $F$ into the formula for the Kähler potential (26), compute the metric and compare with the known form of the Atiyah-Hitchin metric:

$$
d s^{2}=d \kappa^{2}+a^{2} \sigma_{1}^{2}+b^{2} \sigma_{2}^{2}+c^{2} \sigma_{3}^{2}
$$

where

$$
a b=-2 E K+2 k^{\prime 2} K^{2}, \quad b c=-2 E K, \quad c a=-2 E K+2 K^{2},
$$

and $k^{2}+{k^{\prime}}^{2}=1, K^{\prime}(k)=K\left(k^{\prime}\right), d \kappa=-(a b c) d\left(K^{\prime} / \pi K\right)$, and $E$ is the complete elliptic integral of second kind. Here, $\sigma_{1}, \sigma_{2}, \sigma_{3}$ are the forms invariant with respect to the $S U(2)$ generated by the vector fields (55). This direct computation, however, is quite messy and we instead checked that the complex structure $\mathbf{I}$ and the Kähler form $\omega_{1}$ as computed from the generalized Legendre transform construction coincide with the known ones: $z$ is clearly a holomorphic coordinate and a lengthy calculation shows that $u=F_{v}$ is holomorphic with respect to the known complex structure $\mathbf{I}$ as well. The Kähler form $\omega_{1}=d z \wedge d\left(F_{z}\right)+d v \wedge d\left(F_{v}\right)$ is easy to compute. In a basis $d \kappa, d \theta, d \phi, d \psi$, its components are:

$$
\begin{aligned}
& \omega_{1}[1,2]=(b-a) \sin (\psi) \cos (\psi) \sin (\theta), \\
& \omega_{1}[1,3]=a \sin ^{2}(\theta) \cos ^{2}(\psi)+b \sin ^{2}(\theta) \sin ^{2}(\psi)+c \cos ^{2}(\theta) \\
& \omega_{1}[1,4]=c \cos (\theta) \\
& \omega_{1}[2,3]=\left(-a b+a c \sin ^{2}(\psi)+b c \cos ^{2}(\psi)\right) \sin (\theta) \cos (\theta) \\
& \omega_{1}[2,4]=c\left(a \sin ^{2}(\psi)+b \cos ^{2}(\psi)\right) \sin (\theta) \\
& \omega_{1}[3,4]=(b-a) c \sin (\psi) \cos (\psi) \sin ^{2}(\theta) .
\end{aligned}
$$

This is precisely the Kähler form of the Atiyah-Hitchin metric.

\section{More examples, conjectures and conclusions.}

Given a function $G(\eta, \zeta)$, the (generalized) Legendre transform construction produces a hyperkähler metric. Unfortunately, this technique is purely local; it is not clear how to address the issue of geodesic completeness in general. 
We notice, however, that for many known complete metrics the function $G(\eta, \zeta)$ has a very simple form: it is a superposition of the same terms at different mass points. For example, in the class of metrics with triholomorphic isometry (so that the twistor space projects onto $\mathcal{O}(2)$ ) the $A_{k} A L E$-metrics are constructed out of a function $G$ that is a superposition at different "mass" points of the following basic block: $G_{0}=\eta(\ln (\eta)-1)$. A function $G$ that consists of just one term like this gives flat space. Another form of $G$ that corresponds to flat space is $G_{n u t}=\eta^{2} / \zeta$. It is known [2] that adding a $G_{N U T^{-}}$ term to superposition of $G_{0}$ terms gives a complete metric of Taub-NUT type. In particular, for $G=G_{0}+m G_{N U T}$, the Legendre transform construction gives the Taub-NUT metric with mass parameter $m$ (44).

Next let us consider metrics whose twistor space projects on $\mathcal{O}(4)$. The function $G$ in the case considered section six consists of two parts $\tilde{G}_{N U T}=$ $\eta / \zeta$ and $\tilde{G}_{0}=\sqrt{\eta}$. The Atiyah-Hitchin metric asymptotically approaches the Taub-NUT metric (for $m=-1$ ). In this limit $\eta_{A H}=\eta_{\text {Taub-NUT }}^{2}$, and the twoparts of the function $G$ go to $G_{0}$ and $G_{N U T}$ of the Taub-NUT metric (the $\ln (\eta)$ in $G_{0}$ serves merely to define the contour of integration, and doesn't appear in the Atiyah-Hitchin case). We conjecture that superimposing terms of the type $\tilde{G}_{0}$ and $\tilde{G}_{N U T}$ gives new complete metrics.

There are also descriptions of flat space whose twistor space naturally projects on $\mathcal{O}(4)$. These have not been used to construct complete hyperkähler metrics, but are obvious candidate ingredients. They are $G=$ $\eta^{2} / \zeta^{3}$ and $G=\eta(\ln (\eta)-1) / \zeta$ where in the latter case, the contour for the $\ln (\eta)$ term encloses pairs of the roots of $\eta$ as in contour $C_{2}$ of Figure 1.

As another example, we conjecture that the expression

$$
G(\eta, \zeta)=\sum_{i=1}^{k}\left(\left(\sqrt{\eta}-a_{i}\right) \ln \left(\sqrt{\eta}-a_{i}\right)+\left(\sqrt{\eta}+a_{i}\right) \ln \left(\sqrt{\eta}+a_{i}\right)\right)
$$

is related to $D_{k} A L E$-metrics [12]. Work on this conjecture is in progress [12]. The addition of the term $\widetilde{G}_{N U T}$ is interesting, and in particular the metric with $k=2$ has been considered by Hitchin as a degenerate case of K3 [9].

Finally, we make a few comments about higher dimensional hyperkähler metrics. In the $\mathcal{O}(2)$ case, examples based on superpositions of $G_{0}$ and $G_{N U T}$ involving several independent multiplets $\eta_{i}$ were given in [1]. Recently, Gibbons and Manton have used such a metric to describe the asymptotic 
form of the metric on multi-monopole moduli spaces [13]; it is tempting to speculate that a similar expression using our description of the AtiyahHitchin metric may give at least a better approximation.

\section{Acknowledgements}

We are indebted to Claude LeBrun for the numerous discussions from which we benefited greatly. We also thank D. Jatkar for discussions in the early stages of this project, and J. de Boer for carefully reading the manuscript. This work is supported in part by NSF Grant no. PHY9309888.

\section{References}

[1] U. Lindstrom and M. Roček, Nucl. Phys. B222 (1983) 285.

[2] N. J. Hitchin, A. Karlhede, U. Lindstrom, M. Roček, Commun. Math. Phys. 108 (1987) 535.

[3] A. Karlhede, U. Lindstrom, M. Roček, Commun. Math. Phys. 108 (1987) 529.

[4] U. Lindstrom and M. Roček, Commun. Math. Phys. 115 (1988) 21.

[5] A. Besse, Einstein Manifolds., Berlin (1987).

[6] K. P. Tod and R. S. Ward, Proc. R. Soc. Lond. A 368 (1979) 411.

[7] M. Atiyah and N. Hitchin, The geometry and dynamics of magnetic monopoles., Princeton, NJ (1988).

[8] N.J. Hitchin, Math. Proc. Camb. Phil. Soc. 83 (1979) 465.

[9] N.J. Hitchin, in Global Riemannian geometry, T.J. Willmore and N.J. Hitchin Eds., Chichester (1984).

[10] A. Erdelyi, Ed., Bateman Manuscript Project, vol. II, Higher Transcedental Functions., New York (1953). 
[11] I.M. Gelfand, R.A. Minlos and Z.Y. Shapiro, Representations of the rotation and Lorentz groups and their application., New York (1963).

[12] I. T. Ivanov, in preparation.

[13] G.W. Gibbons and N.S. Manton, Phys. Lett. B 356 (1995) 32. 